\title{
SYMMETRIC FUNCTIONS: A BIJECTIVE IDENTITY
}

\section{N. METROPOLIS AND GIAN-CARLO ROTA}

(Communicated by Thomas H. Brylawski)

\begin{abstract}
We give a bijective proof of a classical identity which we have named the cyclotomic identity.
\end{abstract}

1. Introduction. One of the present tasks of combinatorics is the formulation of a bijective theory of symmetric functions. The original program of such a theory was to replace every numerical identity among symmetric functions by an explicit bijective identity among sets. We find that such a program must be enlarged to include bijective identities among multisets as well. In fact, the gist of the identity we give below is the interaction between sets and multisets, an interaction which we are still a long way from fully understanding. This program has been pursued by several authors.

In this note we present a very small contribution to this program by giving a bijective analog and interpretation of the following classical indentities:

$$
\sum_{i=0}^{n}(-1)^{i} a_{i}\left(x_{1}, x_{2}, \ldots\right) h_{n-i}\left(x_{1}, x_{2}, \ldots\right)=0 \text { for } n>0
$$

and $a_{0}\left(x_{1}, x_{2}, \ldots\right) h_{0}\left(x_{1}, x_{2}, \ldots\right)=1$, where

$$
a_{k}\left(x_{1}, x_{2}, \ldots\right)=\sum_{i_{1}<i_{2}<\cdots<i_{k}} x_{i_{1}} x_{i_{2}} \cdots x_{i_{k}}
$$

and

$$
h_{k}\left(x_{1}, x_{2}, \ldots\right)=\sum_{i_{1} \leq i_{2} \leq \cdots \leq i_{k}} x_{i_{1}} x_{i_{2}} \cdots x_{i_{k}}
$$

and where we set $a_{0}\left(x_{1}, x_{2}, \ldots\right)=h_{0}\left(x_{1}, x_{2}, \ldots\right)=1$.

$a_{k}$ and $h_{k}$ are respectively the $k$ th elementary symmetric function and the $k$ th complete symmetric function $[2,3]$.

The classical proof of the identities depends on one simple fact, namely, the identity

$$
\left(\prod_{i}\left(1-t x_{i}\right)\right) \prod_{j}\left(1-t x_{j}\right)^{-1}=1 .
$$

Our proof gives a bijective interpretation of this fact.

Received by the editors January 24, 1986.

1980 Mathematics Subject Classification (1985 Revision). Primary 05A19.

Partially supported by NSF Grant MCS 8104855 . 
2. Proof of the identity. When $E_{1}, E_{2}$ is an ordered pair of subsets of a set $S$ such that $E_{1} \cap E_{2}=\varnothing$ and $E_{1} \cup E_{2}=S$, we write $E_{1}+E_{2}=S$ (either $E_{1}$ or $E_{2}$ may be the empty set).

Let $A_{1}$ be a set whose elements are functions from $E_{1}$ to a set $X$, and $A_{2}$ a set whose elements are functions from $E_{2}$ to $X$. We write $A_{1} A_{2}$ to denote the set of all functions $f: S \rightarrow X$ such that the restrictions satisfy the following conditions:

$$
\left.f\right|_{E_{1}} \in A_{1},\left.\quad f\right|_{E_{2}} \in A_{2} \text {. }
$$

Let $a(S \mid X)$ be the set of all injective functions $S \rightarrow X$, and let $h(S \mid X)$ be the set of all dispositions $S \rightarrow X$.

We recall that a disposition $S \rightarrow X$ is a function $f: S \rightarrow X$ together with a linear order on each fiber $f^{-1}(x), x \in X$, whenever $f^{-1}(x) \neq \varnothing$ (recall that there is exactly one linear order on the empty set).

Our objective is to prove the set-theoretical identity

$$
\sum_{E_{1}+E_{2}=S}(-1)^{\left|E_{1}\right|} a\left(E_{1} \mid X\right) h\left(E_{2} \mid X\right)=0
$$

if $|S|>0$. Clearly, we also have $a(\varnothing \mid X) h(\varnothing \mid X)=1$, where 1 denotes the unique function from the empty set to $X$.

It is clear that identities $(*)$ and $(* *)$ are related. Of the two, $(* *)$ is the stronger identity, since it relates directly to sets and multisets. It is easy to derive (*) from $(* *)$ by defining a suitable notion of "generating function." We shall leave such a proof as an exercise for the reader, since to give it in full would be contrary to the spirit of the present paper.

One important remark is in order, regarding the interpretation of the minus signs in (**). Identity $(* *)$ is an identity among multisets. Now, a multiset $m$ on a set $A$ is a function $m$ from $A$ to the nonnegative integers. The set of such functions forms an Abelian group, and identity (**) holds in this Abelian group. The 0 on the right side of $(* *)$ stands for the identically zero function.

For the proof of $(* *)$, we begin by remarking that if $Y, Z$ are disjoint subsets of $X$, one verifies by simple combinatorial arguments that

$$
a(S \mid Y+Z)=\sum_{E_{1}+E_{2}=S} a\left(E_{1} \mid Y\right) a\left(E_{2} \mid Z\right)
$$

and

$$
h(S \mid Y+Z)=\sum_{E_{1}+E_{2}=S} h\left(E_{1} \mid Y\right) h\left(E_{2} \mid Z\right) .
$$

In the proof, we may assume $X$ is finite. We proceed by induction on $|X|$.

For $|X|=1$, we have $a(S \mid X)=\varnothing$ if $|S|>1$, and the identity to be proved reduces to

$$
a(\varnothing \mid X) h(S \mid X)-\sum_{\substack{E_{1}+E_{2}=S \\\left|E_{1}\right|=1}} a\left(E_{1} \mid X\right) h\left(E_{2} \mid X\right)=\varnothing .
$$

But $a(\varnothing \mid X) h(S \mid X)=h(S \mid X)$; therefore the identity to be proved is

$$
h(S \mid X)=\sum_{\substack{E_{1}+E_{2}=S \\\left|E_{1}\right|=1}} a\left(E_{1} \mid X\right) h\left(E_{2} \mid X\right)
$$


which is trivial. (Note that an injective function is also an injective disposition, and conversely.)

For the general case, let $Y \subset X, Z \subset X, Y \cap Z=\varnothing, Y+Z=X, Y \neq \varnothing$, $Z \neq \varnothing$. We may assume by the induction hypothesis that (**) holds when $X$ is replaced by either $Y$ or $Z$.

The identity to be proved is

$$
\sum_{E_{1}+E_{2}=S}(-1)^{\left|E_{1}\right|} a\left(E_{1} \mid Y+Z\right) h\left(E_{2} \mid Y+Z\right)=\varnothing .
$$

Now, as remarked above,

$$
a\left(E_{1} \mid Y+Z\right)=\sum_{E_{11}+E_{12}=E_{1}} a\left(E_{11} \mid Y\right) a\left(E_{12} \mid Z\right)
$$

and

$$
h\left(E_{2} \mid Y+Z\right)=\sum_{E_{21}+E_{22}=E_{2}} h\left(E_{21} \mid Y\right) h\left(E_{22} \mid Z\right) .
$$

Substituting in $(* *)$ we obtain

$$
\sum_{E_{1}+E_{2}=S}(-1)^{\left|E_{1}\right|} \sum_{E_{11}+E_{12}=E_{1}} \sum_{E_{21}+E_{22}=E_{2}} a\left(E_{11} \mid Y\right) a\left(E_{12} \mid Z\right) h\left(E_{21} \mid Y\right) h\left(E_{22} \mid Z\right) .
$$

Setting $E_{11}=F_{11} ; E_{21}=F_{12}, E_{12}=F_{21} ; E_{22}=F_{22}$, this sum can be rewritten as

$$
\sum_{F_{1}+F_{2}=S} \sum_{F_{11}+F_{12}=F_{1}} \sum_{F_{21}+F_{22}=F_{2}}(-1)^{\left|F_{11}\right|+\left|F_{21}\right|} a\left(F_{11} \mid Y\right) a\left(F_{21} \mid Z\right) h\left(F_{12} \mid Y\right) h\left(F_{22} \mid Z\right)
$$

Changing the order of summation, we obtain

$$
\begin{gathered}
\sum_{F_{1}+F_{2}=S}\left(\sum_{F_{11}+F_{12}=F_{1}}(-1)^{\left|F_{11}\right|} a\left(F_{11} \mid Y\right) h\left(F_{12} \mid Y\right)\right) \\
\cdot\left(\sum_{F_{21}+F_{22}=F_{2}}(-1)^{\left|F_{21}\right|} a\left(F_{21} \mid Z\right) h\left(F_{22} \mid Z\right)\right) .
\end{gathered}
$$

By the induction hypothesis, at least one of the terms in parentheses is always zero if $|S|>0$, therefore the whole expression equals $\varnothing$, and the proof is complete.

\section{REFERENCES}

1. P. Doubilet, On the foundations of combinatorial theory. VII: Symmetric functions through the theory of distribution and occupancy, Stud. Appl. Math. 51 (1972), 377-396.

2. P. A. MacMahon, Combinatory analysis. I, Chelsea, New York, 1960.

3. I. G. MacDonald, Symmetric functions and Hall polynomials, Clarendon Press, Oxford, 1979.

Theoretical Division, los alamos national laboratory, los alamos, New MEXICO 87545

Department of Mathematics, Massachusetts institute of Technology, CAMBRIDGE, MASSACHUSETTS 02139 